\title{
Benign bronchoesophageal fistula: Report of four cases
}

\author{
Salvatore Griffo, MD, ${ }^{a}$ Paolo Stassano, MD, ${ }^{\text {b }}$ Gabriele lannelli, MD, ${ }^{\text {b }}$ Luigi Di Tommaso, MD, ${ }^{\mathrm{b}}$ Marcellino Cicalese, MD, \\ Mario Monaco, MD, ${ }^{b}$ and Giovanni Ferrante, MD, ${ }^{a}$ Naples, Italy
}

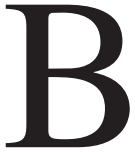

enign bronchoesophageal fistula (BEF) is rare and may be characterized by nonspecific symptoms that may delay a correct diagnosis. ${ }^{1}$ As soon the diagnosis of $\mathrm{BEF}$ is made, immediate surgical treatment is necessary to avoid complications. We present 4 cases of benign BEF that we encountered over a 20 -year period.

\section{Clinical Summary}

Four patients, 2 male and 2 female, mean age $44.0 \pm 25.2$ years, underwent surgery for BEF. Clinical and diagnostic characteristics are in Table 1. The initial symptomatology was elusive in all patients: recurrent pulmonary infections, mild dysphagia, and asthmalike crises. These symptoms lasted for several months before the appearance of coughing on liquid ingestion, which is diagnostic for BEF. Plain chest radiography and computerized tomographic (CT) scan showed an irregular mass in the lung fields but were not

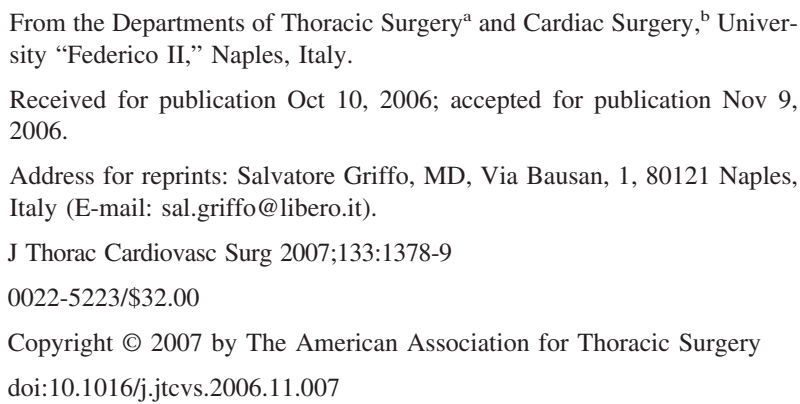

decisive in the diagnosis of BEF. Bronchoscopy and esophagoscopy were not always diagnostic, frequently showing only inflammation in the esophagus or bronchus, or both. The definitive diagnostic tool was esophagography with leakage of contrast medium into the bronchus.

Surgery was carried out through a thoracotomy in 3 patients (left in 1 patient and right in 2 patients) and through a cervicotomy in 1 patient. The BEF was excised, and bronchial and esophageal openings were closed with interrupted sutures. A pleural flap was added in 3 patients. Additional surgery, as a consequence of BEF, was required in 3 patients, and a left pneumonectomy, because of bronchial carcinoma, was carried out in another patient. Pathologic examination showed congenital origin in 1 patient, in whom the bronchus and the esophagus were connected through a bronchogenic cyst (Figure 1). Tubercular infection caused the BEF in 2 patients, and trauma was the cause in a young girl who was struck in the neck by her bicycle handlebars.

At a mean follow-up of $12 \pm 5.6$ years (range 7-20 years), all patients are in good health with no BEF recurrence.

\section{Discussion}

Benign BEFs are rare. They can be congenital or acquired. Congenital BEFs, classified by Braimbridge and Heith, ${ }^{2}$ result from failed tracheoesophageal separation in the early stage of embryonic development and, if they are not associated with esophageal atresia, they can be silent for many years until symptoms appear. ${ }^{1-3}$ A bronchogenic cyst, as in our third patient, is sometime present in congenital BEF. An epithelium-lined tract with muscularis muco-

TABLE 1. Clinical and diagnostic characteristics

\begin{tabular}{|c|c|c|c|c|}
\hline & Patient 1 & Patient 2 & Patient 3 & Patient 4 \\
\hline Age (y) & 45 & 8 & 61 & 62 \\
\hline Sex & Male & Female & Male & Female \\
\hline Clinical diagnosis & Likely & Suspect & Likely & Likely \\
\hline Chest x-ray film & Negative & Negative & Negative & Negative \\
\hline \multicolumn{5}{|l|}{ Chest CT scan } \\
\hline Plain & Negative & Suspect & Negative & Suspect \\
\hline $\begin{array}{l}\text { After esophagogram with } \\
\text { contrast medium }\end{array}$ & Suspect & Diagnostic & Negative & Diagnostic \\
\hline Esophagography & Diagnostic & Negative & Diagnostic & Diagnostic \\
\hline \multicolumn{5}{|l|}{ Endoscopy } \\
\hline Bronchial & Negative & Negative & Negative & Negative \\
\hline Esophageal & Suspect & Negative & Diagnostic & Diagnostic \\
\hline Bronchial location & Right main stem & Right upper & Right lower & Right lower \\
\hline Esophageal location & Medium & Proximal & Distal & Medium \\
\hline Etiology & Infective & Traumatic & Congenital & Infective \\
\hline Surgery & Suture + pedicled flap & Direct suture & Suture + pedicled flap & Suture + pedicled flap \\
\hline Additional surgery & Left pneumonectomy* & Lobectomy & Lobectomy & Segmentectomy \\
\hline
\end{tabular}

$C T$, Computed tomographic. *Because of lung cancer. 


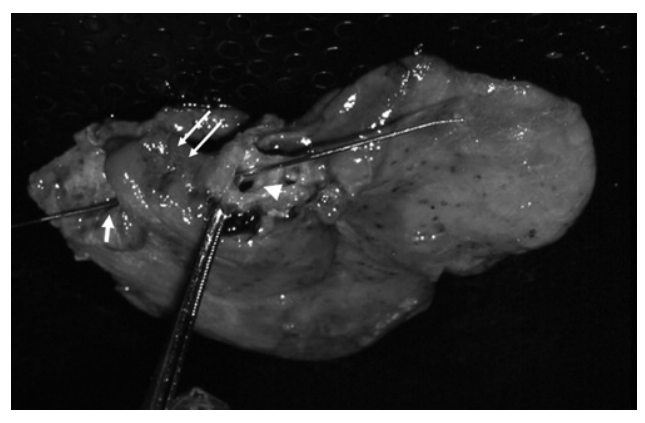

Figure 1. The probe connects the esophageal (arrowhead) and bronchial (arrow) openings through a bronchogenic cyst (double arrows).

sae and the absence of relevant inflammation around the fistula are conclusive for congenital BEF. Acquired BEFs are more common than congenital types and can be of traumatic, inflammatory, infective, and iatrogenic origin. They may appear at any time during the life span. ${ }^{1-4}$

Over a long period of time, patients with BEF may have nonspecific signs such as vague thoracic or epigastric pain, heartburn, mild dysphagia, weight loss, and recurrent pulmonary infections. Coughing after liquid ingestion is a more specific sign, frequently associated with productive sputum, fever, and hemoptysis. Usually symptoms depend on the fistula size, being more evident in short, wide communications and more discrete in narrow fistulas.

A wide array of diagnostic tools is available in the diagnosis of BEF (Table 1). Plain radiography and CT scan of the chest give information on the lungs and mediastinum, but they may not confirm the clinical suspicion. Endoscopic examinations of bronchi and esophagus may fail to identify the anomalous orifice but may reveal inflammatory changes, may help in the surgical approach, as in our second patient, and may exclude a malignancy. ${ }^{1-4}$ Esophagography with dilute barium is almost always decisive in the diagnosis of BEF even if sometimes, despite thorough investigation, the diagnosis is made only at surgery. Once diagnosed, benign BEFs require immediate surgical treatment because, if left untreated, they may pose risks of chronic sepsis, airway and lung tissue destruction requiring additional surgery (as was the case in 3 of our patients), and fatal massive hemoptysis.

The principles for successful fistula management are as follows: control of sepsis, establishment of a good nutritional status, pulmonary support and rehabilitation, and surgical repair. Through a thoracotomy, direct suturing or excision of the BEF with interposition of a pedicled flap is the preferred method of treatment. Cervical approach, as in our second patient, should be reserved for higher BEFs, which are indeed very uncommon. Additional lung segmentectomy or lobectomy is necessary when lung parenchyma is involved. Endoscopic treatment and even medical treatment alone have also been described. ${ }^{5}$

In conclusion, a high index of suspicion in every patient with chronic pulmonary infections of obscure etiology is important in establishing a correct diagnosis. Prompt surgical repair gives the best chances of success.

\section{References}

1. Mangi AA, Gaissert HA, Wright CD, Allan JS, Wain JC, et al. Benign broncho-esophageal fistula in the adult. Ann Thorac Surg. 2002;73: 911-5.

2. Braimbridge MV, Heith HI. Oesophago-bronchial fistula in the adult. Thorax. 1965;20:226-33.

3. Rämö OJ, Salo JA, Mattila SP. Congenital bronchoesophageal fistula in the adult. Ann Thorac Surg. 1995;59:887-90.

4. Cherveniakov A, Tzekov C, Grigorov GE, Cherveniakov P. Acquired benign esophago-airway fistulas. Eur J Cardiothorac Surg. 1996;10: 713-6.

5. Marco C, Doncel F, Veloso E, Viver JM, Vidal J. Non surgical closure of a benign oesophagobronchial fistula. Br J Surg. 1986;74:415. 